\title{
Fibrinogen Apheresis and Peripheral Vascular Disease: Some Progress?
}

\author{
Helmut Schiffl \\ University of Munich, Department of Internal Medicine, Munich, Germany
}

Sir,

Ramunni et al. [1] showed that a single session of selective fibrinogen apheresis reduced circulating fibrinogen by $50 \%$. They failed to document significant changes in microvascular haemodynamics by laser Doppler flowmetry in their patients affected by various stages of peripheral occlusive arterial disease. Of clinical relevance, symptomatic improvement was impressive, with both total and pain-free walking distance increasing significantly.

Short-term improvement in delayed wound healing, another clinical symptom of severe impairment of microcirculation, has been reported for few patients with diabetic foot syndrome or patients with ischemic skin lesions [2]. Our group performed repetitive treatments with selective fibrinogen adsorption (TheraSorb Technology, Plasma Select AG, Teterow, Germany) for 4 weeks in 4 diabetic patients [3]. Treatments were scheduled on days $1,2,4,6,8$, $10,13,16,19,22,25$, and 28 . No clinical side effects of the procedure were observed during 46 sessions. Multiple treatments resulted in a more pronounced depletion of fibrinogen levels (up to $70 \%$ ) than a single session. Both whole blood and plasma viscosity were significantly improved by repeated aphereses. During combined fibrinogen apheresis and specialized multimodal wound care 3 out of 4 patients showed improved wound healing. After termination of apheresis 1 patient underwent surgery for diabetic foot syndrome. These effects of selective fibrinogen apheresis on microvascular haemodynamics may relate not only to sustained lowering of fibrinogen, but also to sustained reduction in the levels of low-density lipoprotein cholesterol, C-reactive protein and adhesion molecules.

There is an urgent need for prospective randomized investigations with selective fibrinogen apheresis in diseases with altered microvascular haemodynamics to corroborate the potential beneficial acute or short-term effects observed in small pilot studies. Moreover, prospective comparisons of this apheresis device with non-se- lective plasma exchange or low-density lipoprotein apheresis are lacking. This places considerable constraints on a more widespread application of the costly and timeconsuming selective fibrinogen apheresis.

\section{References}

1 Ramunni A, Brescia P, Quaranta D, Plantamura M, Ria R, Coratelli P: Fibrinogen apheresis in the treatment of peripheral arterial disease. Blood Purif 2007;25:404-410.

2 Richter WO, Schneidewind JM, Ramlow W, Jahn P, Jung N, Koll R, Klinkmann J: Extracorporeal fibrinogen adsorption - Efficacy, selectivity and safety in healthy subjects and patients with foot ulcers. Transfus Apher Sci 2002;26:15-27.

3 Hierl FX, Petersen A, Landgraf R, Schiffl H, Spannagl M: First results of selective fibrinogen elimination by Rheosorb absorbers in the treatment of the diabetic foot syndrome. Int Symp 'Fibrinogen as a determinant of blood flow properties. Safety and efficacy of fibrinogen lowering with the RheoSorb system', Berlin, January 2000.

\section{KARGER \\ Fax +41613061234 \\ E-Mail karger@karger.ch}

www.karger.com (c) 2008 S. Karger AG, Basel

0253-5068/07/0256-0515\$23.50/0

Accessible online at:

www.karger.com/bpu
Prof. Dr. H. Schiffl

University of Munich, Department of Internal Medicine

Ziemssenstrasse 1

DE-80336 Munich (Germany)

Tel. +49 895160 8397, Fax +49 895705 727, E-Mail hschiffl@hotmail.com 


\section{Reply}

\section{Alfonso Ramunni}

Division of Nephrology, Department of Internal and Public Medicine, University of Bari, Bari, Italy

Sir,

I agree with the author of the letter on the necessity of carrying out multicenter studies, numerically suitable, with the objective of confirming the efficiency of apheresis procedures in the treatment of microcirculation disorders.

However, I do not think that the use of highly selective apheresis, such as fibrinogen apheresis, should be considered an expensive or time-consuming event. On the contrary, the possibility of withdrawing specific molecules from the bloodstream allows for the capacity to investigate the pathological process at the base of the vascular damage, which is certainly precluded from the plasma exchange, whose com- plete absence of selectivity in the elimination of substances does not provide any useful information on the matter. In our article, to which the author of the letter refers, our aim has been to investigate, among other factors, some markers of the endothelial activation.

One possibility in this sense also derives from double filtration plasmapheresis, a semi-selective procedure to use and compare with decidedly selective apheresis in the treatment of peripheral arterial disease. Only the comparison between the efficiency of low-density lipoprotein apheresis and fibrinogen apheresis on the one hand and double filtration plasma- pheresis on the other will allow us to better understand the pathogenesis of microcirculation disturbances, helping us to choose the most efficient procedure for the treatment of our patients. In this view, the methodologies of selective apheresis, besides the strategies of a therapeutic approach, are to be considered real laboratories of in vivo investigation, which help us to understand the role of individual molecules in the development of vascular pathologies. Consequently, instead of being dismissed for the costs or workload, they should be used on a greater scale, with the objective of clarifying their correct utilization. 